\title{
A SWITCHED DYNAMIC MODEL FOR POINTING TASKS WITH A COMPUTER MOUSE
}

\author{
STANISLAV ARANOVSKIY, ROSANE USHIROBIRA, DENIS EFIMOV, AND GÉRY \\ CASIEZ
}

\begin{abstract}
In this paper, we study one of the most fundamental tasks in human-computer interaction, the pointing task. It can be described simply as reaching a target with a cursor starting from an initial position (e.g. executing a movement using a computer mouse to select an icon). In this paper, a switched dynamic model is proposed to handle cursor movements in indirect pointing tasks. The model contains a ballistic movement phase governed by a nonlinear model in Lurie form and a corrective movement phase described by a linear visual-feedback system. The stability of the model is first established and the derived model is then validated with experimental data acquired in a pointing task with a mouse. It is established that the measured data of pointing movements of different types can be fitted within the proposed model. Numerical comparison against pointing models available in the literature is also provided.
\end{abstract}

\section{INTRODUCTION}

The domain of human-computer interaction (HCI) covers a great deal of different tasks that can be performed by a user, the pointing task being one of the most frequently executed. In a graphical interface of any interactive system, the task of pointing for a user consists in displacing the pointer from a starting point to a final point in order to select a target (e.g. for instance, to select another window). It can be perceived and executed in different ways, depending on the used system, and the interaction may be direct or indirect. In direct interactions, users interact with a touch screen by moving hands and fingers, this input follows then different layers of hardware and software processes to produce the final visual response on the same screen. Indirect interactions deal with human inputs coming from an independent device, such as a computer mouse or a touchpad, based on visual information on a screen, that go through similar processes as in the direct interactions, ending with a visual response on the same screen. In the case of direct interactions the pointer is displayed on the same screen where the user interaction takes place while in the case of indirect interactions, the user controls the pointer displayed on a screen by the means of a different tool.

The pointing transfer function (PTF) corresponding to the pointing task is characterized by the ratio of the amount of movement of an input device (e.g. a mouse) and the controlled object (e.g. a cursor). In early graphical user interfaces, PTF were typically chosen as a constant control-to-display (CD) gain, while modern

Key words and phrases. Pointing motion, modeling, human-computer interaction, switched model, pointing transfer function.

This work was supported by ANR (TurboTouch, ANR-14- CE24-0009). Stanislav Aranovskiy is supported by the Russian Science Foundation grant (project 17-79-20341). 
graphical user interfaces, such as Windows, OS X, and Xorg, track the input device velocity and dynamically adjust the CD gain over time [1]. The idea behind such an adjustment is to provide small amplification for low input velocities to improve pointing accuracy, and to provide high amplification for high input velocities to reduce traveling time. Experimental studies [2] report that the choice of a PTF affects human-computer interaction performance, and users get better performance using switching PTF. However, despite all the research on evaluation and reverse-engineering of the existing PTF, the problem of PTF design and optimization continues to exist since there are no actual dynamical models for human pointing.

Models of pointing dynamics would also be of interest for endpoint prediction techniques [3], where the system attempts to predict the target cursor position from the beginning of the pointing movement. Such a prediction is further used to dynamically modify the visual interface (e.g. to reduce the distance to the target or increase its width). Some techniques and methods can be found in [4], and a toolbar with dynamically expanding icons represents an example of such an approach [5]. Some other challenging applications where dynamic models of (pointing) movements can be relevant include analyses of mouse movements for user identification [6], in robotics for motion planning [7,8] and for human movement estimation [9], e.g. for manipulators teleoperation [10].

Models of pointing movements. Models used in the Human-Computer Interaction (HCI) community are mainly focused on static characteristics. They do not describe how exactly a movement is performed or what is its velocity profile, but rather describe static relationships between averaged values: average movement time, target width and traveled distance. The most popular model is the Fitts' law. It was presented in the '50s by Fitts in his seminal paper [11] and claims the following:

$$
M T=a+b I D,
$$

where $M T$ represents the movement time, $a$ and $b$ are positive constants and $I D$ is the index of difficulty of the pointing task. Different formulations exist for the ID but the most commonly used was defined by MacKenzie [12] as follows:

$$
I D:=\log _{2}\left(\frac{D}{W}+1\right)
$$

where $D$ is the distance from the starting point to the target center, and $W$ is the target width. In the next decades, it was found that the relation (1) holds in many experimental studies [12]. One of the first attempts to explain the Fitt's law is the Impulse Variability model proposed by Schmidt et al. [13] that credits almost entirely the motion to an initial muscle impulse.

On the other hand, the Iterative Corrections model proposed in [14] describes the pointing motion as controlled only by a visual feedback. Nevertheless, it was shown that neither of these models takes in consideration all different effects perceived in experiments. The hybrid Optimized Initial Impulse model developed by Meyer et al. [15] is now accepted by the HCI community as the most well-established explanation for Fitts' law [4]. According to Meyer's model, pointing motion can be separated into two distinguishable stages: a rapid and large movement to bring the pointer close enough to the target without visual tracking (ballistic phase), and a 
slower corrective movement to reach the target under feedback control (corrective phase). Moreover, it should be stressed the models in [13-15] do not use differential equations to describe the motion, but mainly determine verbally possible pointing scenarios.

In these days, Fitts' law and the Optimized Initial Impulse model are still the most broadly accepted models of pointing motions within a static point of view. In recent years, most progress in pointing model design are concentrated in extending these two models. For instance, the distribution of $M T$ for computer mouse movements is studied in [16], targeted-tracking tasks are considered in [17], and a model for analyzing rapid point-and-click motions taking into consideration human effects has been proposed recently in [18].

Concerning the ballistic phase, the authors in [19] have observed in experiments that for pointing tasks with low difficulty (i.e. with relatively large values of $W$ ), the target may be selected during the ballistic phase only, and in such cases, the dependence of the movement time $M T$ on the target width $W$ is weak, and $M T$ is mostly proportional to $\sqrt{D}$. This observation also explains why Fitts' law (1) matches poorly experimental data for low values of $I D[20]$.

Many researchers tried to reconstruct the ballistic movement as a solution of an optimal control problem. A well-known result is the minimum jerk model proposed by Hogan in [21], which claims that the ballistic movement is performed in such a way that the total jerk cost along the trajectory is minimized. As it was shown in [22], the minimization of the jerk cost leads to more plausible results than the use of acceleration or snap cost functions. The main drawback of the minimum jerk model is the implied symmetry of the velocity profile that is not often observed in experiments. To this end, a novel discontinuous absolute work cost function has been recently proposed in [23-25] yielding asymmetrical velocity profile. However, this model is applied only to describe vertical arm movements in gravitation field, which is not the case for HCI pointing tasks.

The modeling of the ballistic movement as a sensorimotor feedback control for motor coordination in stochastic environment is discussed in [26,27]. As noted in [27], the magnitude of motor noise is proportional to muscle activation, so minimumvariance models adjust well with the minimum-jerk curves.

To model the tracking stage, the linear time-varying or -invariant models are typically applied. The crossover model [28] is a classic model used in pilot behavior studies and is given by the transfer function

$$
C(s):=K e^{-s T_{0}} \frac{T_{1} s+1}{\left(T_{2} s+1\right)\left(T_{3} s+1\right)},
$$

where $K \geq 0$ and $T_{i} \geq 0, i=0, \ldots, 3$ are model parameters.

Another second-order model resembling a spring-mass-damper system (second order lag) was recently used in [29] to examine pointing dynamics in reciprocal pointing with pointer acceleration.

The goal of the Vector Integration To Endpoint (VITE) model introduced in [30] is to describe a motion controlled by an agonist-antagonist pair of muscles, e.g. wrist rotation. Considering only one muscle, the VITE model is given by

$$
\begin{aligned}
& \dot{P}(t)=G(t)[V(t)]^{+}, \\
& \dot{V}(t)=\gamma(-V(t)+T-P(t)),
\end{aligned}
$$


where $P(t)$ is the pointer position, $V(t)$ is the pointer velocity, $T$ is the target (desired) position, $\gamma>0$ is a constant, $[\cdot]^{+}$denotes a positive projection:

$$
[V]^{+}=\left\{\begin{array}{l}
V \text { for } V \geq 0, \\
0 \text { for } V<0,
\end{array}\right.
$$

and the GO signal $G(t)$ launching the movement execution is a positive constant or a step function.

Assuming that the considered two muscles are equivalent, the projection in (3) can be relaxed, and the VITE model yields a second-order linear time-invariant system, where the stable equilibrium point is $P=T, V=0$. In [31], the authors used a modified version of the VITE model substituting $G(t) \equiv 1$ to analyze the sensorimotor integration in absence of a visual feedback. In robotics, the VITE model can be applied to design human-like pointing movements [7].

The VITE model has been extended quite recently in [32] to study pointing transfer functions. In this paper, the GO signal $G(t)$ in (3) has been substituted by a function of motion acceleration $G(\|V\|)$. In addition, with no feedback delay and a non-decreasing $G(\|V\|)$ function, the closed-loop stability has been proven.

Since for linear systems the transient time is logarithmically related with the traveled distance, then the asymptotic behavior of such models reproduces Fitts' law (1). Nevertheless, these models are only able to describe the corrective phase of the pointing movement, and they cannot model the ballistic part, where the movement time is proportional to the square root of the traveled distance.

To the best of our knowledge, the first attempt to handle both ballistic and tracking phases with a single model was taken almost fifty years ago in [33], and the result is referred to as the Surge model. This model describes the ballistic movement as a time-optimal bang-bang acceleration curve, and the tracking part is governed by the crossover model (2). Switching between these two models occurs when a linear combination of velocity and position error crosses a threshold. The Surge model was applied for a target following task with abrupt jumps of the target, where only qualitative comparison of the model's prediction with the measured data was given. It is also worth noting that the target following task considered in [33] was not in the domain of HCI, thus no PTF was involved in the model design.

As discussed in [34], control theory is a powerful tool to empower the design of computer systems. Particularly, in [35] an experimental comparison of some of the models described above is presented, confirming that control theory offers a promising complement to Fitts' law based approaches in HCI.

Problem statement. In this paper, our objective is the dynamic model identification of pointing motions based on an experimental data acquired with a computer mouse from [1], within a control theory framework. Inspired by the Optimized Initial Impulse model [15] and the Surge model [33], the obtained model would have hybrid or switching nature to represent both ballistic and visual tracking phases. In addition, the model should consider the pointing transfer function (PTF) of the operating system as in [32]. The resulting model can be used to design PTF that may improve the user performance in pointing tasks.

It is worth noting that some types of human movements may be considered as parts of unstable trajectories, e.g. throwing or hitting. However, for pointing motions considered in this paper it is natural to expect a trajectory with smooth velocity acceleration and deceleration at the start and the end of the movement, 
without abrupt changes. This natural considerations confirmed by experimental observations motivate us to search for a stable dynamical model of the pointing movement.

The abridged version of this paper appeared in [36]. The main added values of this submission are: 1) Proposition 1 of this manuscript is stronger than the one of [36]; 2) the proof of Proposition 3 is not given in [36]; 3) significantly extended experimental and numerical results; 4) a comparison against pointing models available in the literature.

The outline of this work has the following structure. In Section 2, the experimental data used in our studies is described. Then in Section 3, the proposed model and an analysis of its properties are given. The results of the experimental validation are illustrated in Section 4. Finally, in the results of experimental validation, conclusions and a discussion of future research directions are provided.

\section{DESCRIPTION OF THE EXPERIMENTAL DATA}

The model proposed in this paper is based on the experiment described in [1], where the authors considered a one-dimensional pointing task and compared the performance of pointing transfer functions used by default in modern operating systems (OS X, Windows and Xorg). To provide a baseline for comparison, a constant CD gain function was also considered by the authors. In this experiment, a 400 counts-per-inch corded USB Logitech mouse was used by the participants to select targets on a 23" display. The targets presented to the participants where solid vertical bars of 4 possible widths: 1 pixel $(0.26 \mathrm{~mm}), 3$ pixels $(0.77 \mathrm{~mm}), 6$ pixels $(1.55 \mathrm{~mm})$, and 9 pixels $(2.32 \mathrm{~mm})$ with the distance between the bars equal to 1163 pixels $(300 \mathrm{~mm})$. More details about the hardware setup and experimental procedure can be found in [1].

To eliminate all uncertainties related to dynamically adjusted PTFs and to focus on modeling human movements, only the static gain PTF is chosen for this paper. Notice that such a static PTF is not used by default by modern operating systems [1], and it has less motion acceleration than a PTF with dynamically adjusted gains. As a result, this choice of a PTF becomes important during the ballistic phase since this phase is not visually tracked. To bring the cursor close to the target position, some participants made a usual wrist-elbow gesture, but then they did not find the cursor where expected due to the less than usually perceived motion acceleration. Thus, they had to perform several ballistic motions before the corrective phase began. As participants adapted their gestures to the constant PTF, the number of repetitive ballistic motions reduced. An example of trajectories of non-trained, partially trained, and trained participants are given in Fig. 1, where multiple ballistic phases are observed for non-trained and partially trained participants. Further, only trained participants will be considered.

\section{MODEL}

3.1. Model overview. The proposed model structure is given in Fig. 2. The dynamics of the pointing device (here, a mouse) is described by the double integrator:

$$
\begin{aligned}
& \dot{P}_{m}(t)=V_{m}(t), \\
& \dot{V}_{m}(t)=A_{m}(t),
\end{aligned}
$$




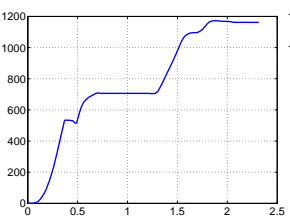

(A)

Not-

trained.

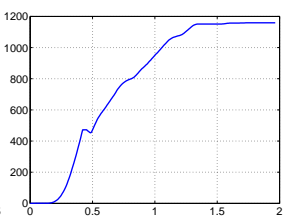

(B)

Par-

tially

trained.

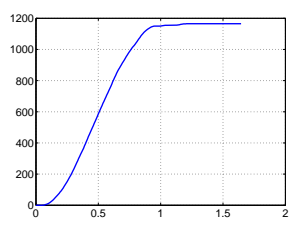

(c) Trained.

Figure 1. Pointing trajectories for differently trained participants, a constant-gain PTF. Cursor position (pixels) versus time (seconds).

where $A_{m}(t)$ is the mouse acceleration, $V_{m}(t)$ is the mouse velocity and $P_{m}(t)$ is the mouse position. The PTF $G(\cdot)$ maps the measured mouse velocity (counts/second) to the cursor velocity $V_{c}(t)$ (pixels/second) as $V_{c}=G\left(V_{m}\right)$.

The following assumption implies that the PTF $G(\cdot)$ belongs to a certain sector $\left[k_{g}, \infty\right)$, where $k_{g}>0$.

Assumption 1. There exists $k_{g}>0$, such that for all $\sigma \neq 0, G(0)=0$ and

$$
\left(G(\sigma)-k_{g} \sigma\right) \sigma>0 \text {. }
$$

Since $k_{g}$ can be chosen arbitrary small, Assumption 1 is not restrictive and is reasonable for practical applications. One possible choice of $G$ is a constant-gain PTF given by

$$
G\left(V_{m}\right):=g_{0} V_{m}
$$

where $g_{0}>0$ is a constant representing both counts-to-pixels scaling and velocity amplification. The differential equation satisfied by the cursor position $P_{c}(t)$ is $\dot{P}_{c}(t)=V_{c}(t)$. The cursor position is visually observed yielding the perceived cursor velocity $V_{\text {per }}(t)$ and the perceived cursor position $P_{p e r}(t)$. The perception process can be described by the stable LTI first-order filter

$$
\begin{aligned}
& V_{p e r}(t)=\frac{1}{\tau_{p e r}}\left(P_{c}(t)-P_{p e r}(t)\right), \\
& \dot{P}_{p e r}(t)=V_{p e r}(t),
\end{aligned}
$$

which is equivalent to

$$
P_{p e r}(t)=\frac{1}{\tau_{p e r} p+1} P_{c}(t) ; \quad V_{p e r}(t)=\frac{p}{\tau_{p e r} p+1} P_{c}(t),
$$




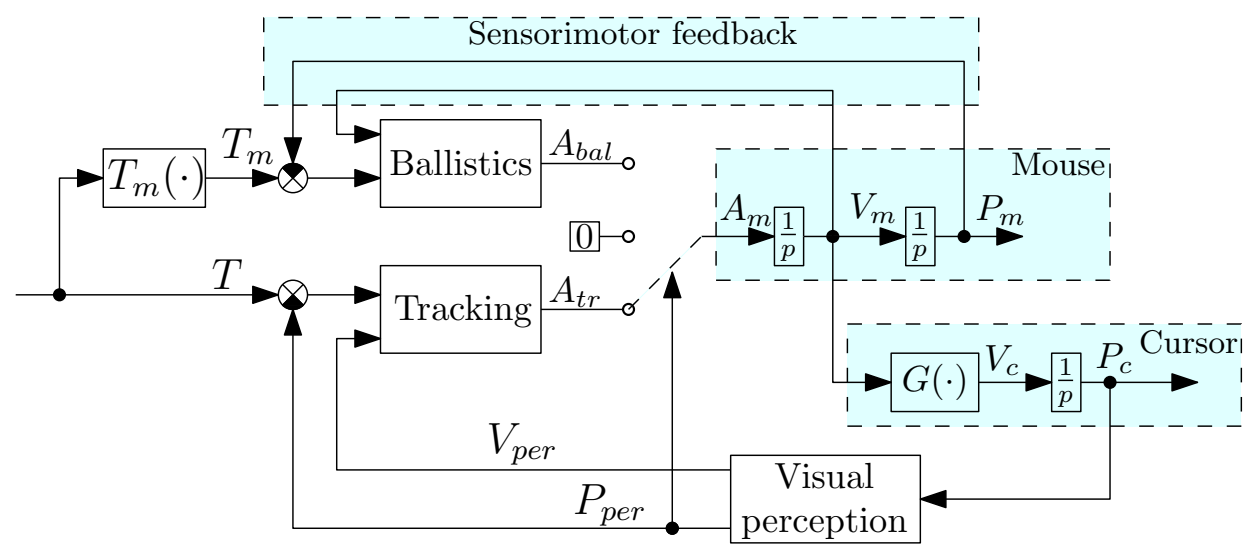

FIGURE 2. The proposed model structure.

where $p=\frac{d}{d t}$ and $\tau_{p e r}>0$ is the perception time.

The observed position $P_{p e r}(t)$ is then used to determine a switching instance of time for the mouse acceleration $A_{m}(t)$. In the beginning of the pointing motion, the movement is in the ballistic phase, and the mouse acceleration $A_{m}(t)$ equals to the accelerations provided by the ballistic dynamics, $A_{b a l}(t)$. When the cursor reaches some predefined position $P_{s w}$ and the participant visually marks it, the motion switches to the corrective phase governed by the visual tracking. This switch is not instantaneous, and there exists the commutation period $\delta_{s w}>0$ when zero acceleration is applied. Define $t_{s w}>0$ as the first instant of time when $P_{p e r}(t)=P_{s w}$. Then

$$
A_{m}(t)= \begin{cases}A_{b a l}(t) & \text { for } t<t_{s w} \\ 0 & \text { for } t_{s w} \leq t \leq t_{s w}+\delta_{s w}, \\ A_{t r}(t) & \text { for } t_{s w}+\delta_{s w}<t\end{cases}
$$

where $A_{t r}(t)$ is the acceleration provided by the tracking dynamics.

The desired cursor position is the input reference signal $T$ (in pixels), where for pointing tasks it is assumed that $T$ is a step function or a constant. Based on the given value of $T$, the desired mouse displacement (in $\mathrm{mm}$ ) is estimated by the participant, $T_{m}=T_{m}(T)$. This mapping is based on the user's experience with the used PTF and it can be approximated as a constant gain,

$$
T_{m}(T):=g_{m} T, \text { with } g_{m}>0 .
$$

When the value $T_{m}$ is set, the ballistic movement is initiated to bring the mouse cursor sufficiently close to the desired position $T$. If the mapping $T_{m}(\cdot)$ is poor, i.e. if the participant does not have sufficient experience with the given PTF, then the resulting ballistic movement may be inaccurate, see Fig. 1a.

Next we will provide accelerations models for the tracking phase $A_{t r}$ and the ballistic phase $A_{b a l}$.

Remark 1. As it is described in Section 2, only trained participants are considered in this study. It is worth to notice that for trained users each motion phase is activated just once, thus, there is no stability issue related to switching for the considered scenario. 
3.2. Tracking dynamics. The tracking dynamics of the proposed model can be seen as an extension of the VITE model (3), (4) taking into account the visual perception (7). Consider

$$
A_{t r}(t):=\gamma\left(-k_{v} V_{p e r}(t)+T-P_{p e r}(t)\right),
$$

with the model parameter $k_{v}>0$. Assume that the model is in the corrective stage, that is $t>t_{s w}+\delta_{s w}$, and combine together the mouse dynamics, the perception, the cursor dynamics, and the tracking law (9). Then the resulting closed-loop system is given by

$$
\begin{aligned}
& \dot{P}_{p e r}(t)=\frac{1}{\tau_{p e r}}\left(P_{c}(t)-P_{p e r}(t)\right), \\
& \dot{P}_{c}(t)=G\left(V_{m}(t)\right), \\
& \dot{V}_{m}(t)=\gamma\left(-\frac{k_{v}}{\tau_{p e r}}\left(P_{c}(t)-P_{p e r}(t)\right)+T-P_{p e r}(t)\right) .
\end{aligned}
$$

Proposition 1. Consider the system (10) with $\gamma>0, k_{v}>\tau_{\text {per }}>0$, and $G(\cdot)$ satisfying Assumption 1. Then the system is globally asymptotically stable with the equilibrium point $P_{\text {per }}=P_{c}=T$ and $V_{m}=0$.

Proof. Define $\tilde{P}_{p e r}(t):=P_{p e r}(t)-T$ and $\tilde{P}_{c}(t):=P_{c}(t)-T$. Then the tracking error model is

$$
\begin{aligned}
& \dot{\tilde{P}}_{c}(t)=G\left(V_{m}(t)\right), \\
& \dot{\tilde{P}}_{p e r}(t)=\frac{1}{\tau_{p e r}}\left(\tilde{P}_{c}(t)-\tilde{P}_{p e r}(t)\right), \\
& \dot{V}_{m}(t)=-\gamma\left(\alpha_{1} \tilde{P}_{c}(t)+\alpha_{2} \tilde{P}_{p e r}(t)\right),
\end{aligned}
$$

where $\alpha_{1}:=\frac{k_{v}}{\tau_{p e r}}$ and $\alpha_{2}:=1-\frac{k_{v}}{\tau_{p e r}}$.

Define $x_{t r}(t):=\left[\begin{array}{lll}V_{m}(t) & -\tilde{P}_{p e r}(t) & -\tilde{P}_{c}(t)\end{array}\right]^{\top}$ and $G^{\dagger}(\sigma):=G(\sigma)-k_{g} \sigma$, and note that $G^{\dagger}$ belongs to the $[0, \infty)$ sector due to (5). The tracking error dynamics (11) can be rewritten as

$$
\begin{aligned}
& \dot{x}_{t r, 1}(t)=\gamma \alpha_{2} x_{t r, 2}(t)+\gamma \alpha_{1} x_{t r, 3}(t), \\
& \dot{x}_{t r, 2}(t)=-\frac{1}{\tau_{p e r}} x_{t r, 2}(t)+\frac{1}{\tau_{p e r}} x_{t r, 3}(t), \\
& \dot{x}_{t r, 3}(t)=-G\left(x_{t r, 1}(t)\right)=-k_{g} x_{t r, 1}(t)-G^{\dagger}\left(x_{t r, 1}(t)\right),
\end{aligned}
$$

that is a nonlinear system in Lurie form,

$$
\begin{aligned}
\dot{x}_{t r}(t) & =A_{s s} x_{t r}(t)+B_{s s} u(t), \\
y(t) & =C_{s s}^{\top} x_{t r}(t), \\
u(t) & =-G^{\dagger}(y(t)),
\end{aligned}
$$

where

$$
\begin{gathered}
A_{s s}:=\left[\begin{array}{ccc}
0 & \gamma \alpha_{2} & \gamma \alpha_{1} \\
0 & -\frac{1}{\tau_{p e r}} & \frac{1}{\tau_{p e r}} \\
-k_{g} & 0 & 0
\end{array}\right], \\
B_{s s}:=\left[\begin{array}{l}
0 \\
0 \\
1
\end{array}\right], C_{s s}:=\left[\begin{array}{l}
1 \\
0 \\
0
\end{array}\right] .
\end{gathered}
$$


The transfer function of the linear part of the system (12) is

$$
\begin{aligned}
W(s) & =C_{s s}^{\top}\left(s I-A_{s s}\right)^{-1} B_{s s} \\
& =\frac{\gamma\left(k_{v} s+1\right)}{\tau_{p e r} s^{3}+s^{2}+k_{g} \gamma k_{v} s+k_{g} \gamma}=: \frac{B_{W}(s)}{A_{W}(s)},
\end{aligned}
$$

and, applying the Routh-Hurwitz stability criterion, $W(s)$ is Hurwitz for $k_{v}>\tau_{p e r}$.

Following the Popov criterion [37], the system (12) is absolutely stable for all $G^{\dagger}$ belonging to the $[0, \infty)$ sector if there exists $\mu>0$, such that

- $-\frac{1}{\mu}$ is not an eigenvalue of $A_{s s}$;

- the transfer function $(\mu s+1) W(s)$ is strictly positive real.

The latter condition is satisfied if for all $\omega \in \mathbb{R}$

$$
\operatorname{Re}[W(\mathrm{i} \omega)]-\mu \omega \operatorname{Im}[W(\mathrm{i} \omega)]>0 .
$$

Since $A_{W}(s)$ is stable, it follows that $\left|A_{W}(\mathrm{i} \omega)\right|^{2}>0$ for all $\omega$, and inequality (13) is replaced with

$$
\operatorname{Re}\left[B_{W}(\mathrm{i} \omega) \overline{A_{W}(\mathrm{i} \omega)}\right]-\mu \omega \operatorname{Im}\left[B_{W}(\mathrm{i} \omega) \overline{A_{W}(\mathrm{i} \omega)}\right]>0,
$$

where $\overline{(\cdot)}$ is the complex conjugate of $(\cdot)$. The inequality (13) is thus equivalent to $\gamma\left(\mu\left(k_{v}-\tau_{p e r}\right)-k_{v} \tau_{p e r}\right) \omega^{4}+\gamma\left(\gamma k_{g} k_{v}^{2}-1\right) \omega^{2}+\gamma^{2} k_{g}>0$. Since $k_{v}-\tau_{p e r}>0$, there exists

$$
\mu_{0}:=\frac{1}{k_{v}-\tau_{\text {per }}}\left(k_{v} \tau_{\text {per }}+\frac{\left(\gamma k_{g} k_{v}^{2}-1\right)^{2}}{4 \gamma k_{g}}\right),
$$

such that the inequality (13) is satisfied for all $\mu>\mu_{0}$. Therefore there exists $\mu>\mu_{0}$ satisfying the conditions of the Popov criterion, and the origin $x=0$ of the system (12) is globally asymptotically stable.

Remark 2. It can be verified that if $G(\cdot)$ is chosen as (6), then the system (10) is stable exponentially.

3.3. Ballistics. In contrast with the tracking phase, the ballistic movement is performed without visual feedback, i.e. the cursor position is not observed by the user in the ballistic phase. However, due to proprioception and sensorimotor integration, users sense the wrist displacement and velocity closing the sensorimotor loop, see Fig. 2.

Study of the available experimental data (see Section 2) shows that a typical ballistic movement of a trained participant can be approximated with the curve given in Fig. 3, where both approximation and measured data are presented.

The acceleration profile of the approximating curve consists of two constant values with a linear transient between them, see Fig. 3c. Note that this ballistic movement is not finished, i.e. the participant's wrist did not stop and the mouse velocity did not reach zero. Indeed, as it is illustrated by Fig. 1a and Fig. 1c, trained participants can notice the cursor (and switch to visual tracking) before finishing the ballistic phase.

The ballistic movement model is proposed as:

$$
\begin{aligned}
& \dot{\tilde{P}}_{m}(t)=V_{m}(t), \\
& \dot{V}_{m}(t)=A_{b a l}(t)=f_{b a l}\left(\tilde{P}_{m}(t), V_{m}(t)\right),
\end{aligned}
$$




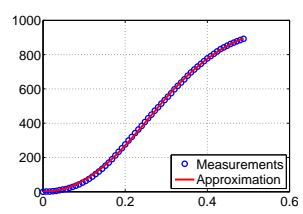

(A)

Po-

si-

tion

(pix-

els)

ver-

sus

time

(sec-

onds).

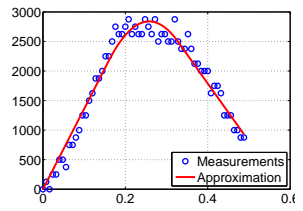

(B)

Ve-

loc-

ity

(pix-

els

per

sec-

ond)

ver-

sus

time

(sec-

onds).

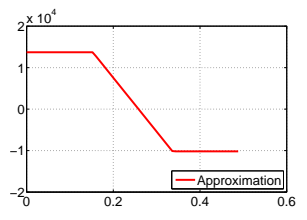

(C)

Ac-

cel-

er-

a-

tion

(pix-

els

per

square

sec-

onds)

ver-

sus

time

(sec-

onds).

Figure 3. Approximation of the measured mouse trajectory for an experienced participant.

where $\tilde{P}_{m}(t):=P_{m}(t)-T_{m}$, and the function $f_{\text {bal }}(\cdot, \cdot)$ is to be defined. The initial conditions are $\tilde{P}_{m}(0)=-T_{m}$ and $V_{m}(0)=0$. 
Define the acceleration profile, Fig. 3c, as

$$
A_{b a l}(t)= \begin{cases}a_{1} & \text { for } t<t_{1}, \\ a_{1}+l\left(t-t_{1}\right) & \text { for } t_{1} \leq t \leq t_{2}, \\ a_{2} & \text { for } t>t_{2},\end{cases}
$$

where $a_{1}, a_{2}$ and $t_{2}>t_{1}>0$ are profile parameters, $a_{1} a_{2}<0$, and $l:=\frac{a_{2}-a_{1}}{t_{2}-t_{1}}$ is the slope of the linear part, $a_{1} l<0$. Define also

$$
\eta(t):=-\tilde{P}_{m}(t)-k_{b a l} V_{m}(t),
$$

where the ballistic model parameter $k_{b a l}>0$. It can be shown via some tiresome but straightforward computations that

$$
k_{b a l}=\frac{2 a_{1}^{3}-6 a_{1}^{2} l t_{1}+3 a_{1} l^{2} t_{1}^{2}-6 l^{2} T_{m}}{3 a_{1} l\left(a_{1}-2 l t_{1}\right)}
$$

ensures that for $t \in\left[t_{1}, t_{2}\right]$ the following holds:

$$
\eta(t)=A_{b a l}(t)\left(p_{2} A_{b a l}^{2}(t)+p_{1} A_{b a l}(t)+p_{0}\right),
$$

where

$$
p_{0}:=\frac{a_{1}\left(a_{1}-2 l t_{1}\right)}{2 l^{2}}, p_{1}:=-\frac{k_{b a l}}{2 l}, p_{2}:=-\frac{1}{6 l^{2}},
$$

and $p_{0}>0, p_{2}<0$. Note that there always exists a value $g_{m}$, see (8), such that $k_{b a l}$ computed as (16) is positive.

To construct the function $f_{\text {bal }}\left(\tilde{P}_{m}, V_{m}\right)$ we want to inverse (17) on the interval $\left[a_{l}, a_{r}\right]$, where $a_{l}:=\min \left(a_{1}, a_{2}\right)<0$ and $a_{r}:=\max \left(a_{1}, a_{2}\right)>0$. Equality (17) defines the implicit function

$$
h\left(A_{b a l}, \eta\right)=A_{b a l}\left(p_{2} A_{b a l}^{2}+p_{1} A_{b a l}+p_{0}\right)-\eta=0 .
$$

Applying the Implicit Function theorem, the desired inversion exists if the partial derivative

$$
\frac{\partial h\left(A_{b a l}, \eta\right)}{\partial A_{b a l}}=3 p_{2} A_{b a l}^{2}+2 p_{1} A_{b a l}+p_{0}
$$

has no root in the interval $\left[a_{l}, a_{r}\right]$. The right-hand side of (19) is a quadratic polynomial with a negative leading coefficient. Its roots are

$$
s_{l, r}:=-k_{b a l} l \pm \sqrt{a_{1}^{2}+k_{b a l}^{2} l^{2}-2 t_{1} a_{1} l} .
$$

Recalling $a_{1} l<0$, it follows that for any value $k_{b a l}$ the roots are real and different; without loss of generality we assume $s_{l}<s_{r}$. The following assumption is instrumental to proceed.

Assumption 2. The parameters $t_{1}, a_{1}, a_{2}$ and $l$ are such that the inequality $t_{1}>$ $\frac{a_{1}^{2}-a_{2}^{2}}{2 a_{1} l}$ holds.

Define

$$
\bar{k}:=\frac{a_{1}^{2}-a_{2}^{2}-2 t_{1} a_{1} l}{2 a_{2} l},
$$

where $\bar{k}>0$ due to Assumption 2. It can be verified that any choice of $0<k_{b a l}<\bar{k}$ yields $s_{l}<a_{l}<0<a_{r}<s_{r}$, i.e. on the interval $\left[a_{l}, a_{r}\right] \subset\left[s_{l}, s_{r}\right], \frac{\partial h\left(A_{b a l}, \eta\right)}{\partial A_{b a l}}>0$ and (17) is invertible. 


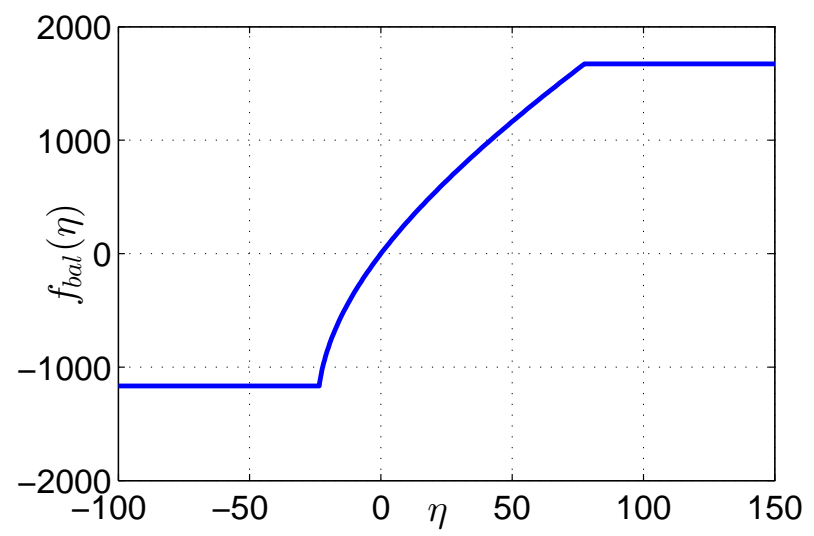

FIgURE 4 . The reconstructed $f_{\text {bal }}(\eta)$ function.

Define the inversion of (17) on the interval $\left[a_{l}, a_{r}\right]$ as $A_{b a l}=\phi(\eta)$, and denote by $\eta_{l}$ and $\eta_{r}$ the solutions of equations $h\left(a_{l}, \eta_{l}\right)=0$ and $h\left(a_{r}, \eta_{r}\right)=0$, respectively. Now we can formulate the following proposition, whose proof follows from the derivations above.

Proposition 2. Let Assumption 2 be satisfied and choose $0<k_{\text {bal }}<\bar{k}$, with $\bar{k}$ as in (20). Then $\phi(\eta)$ for $\eta \in\left[\eta_{l}, \eta_{r}\right]$ is a uniquely defined real-valued monotonic function with $\phi\left(\eta_{l}\right)=a_{l}, \phi(0)=0$ and $\phi\left(\eta_{r}\right)=a_{r}$.

Remark 3. From a practical point of view, both Assumption 2 and the inequality $k_{b a l}<\bar{k}$ are not restrictive. The Assumption 2 implies that the mouse velocity does not return to zero before $t_{2}$. As it is illustrated in Fig. 3, this assumption is reasonable for practical ballistic movements and it was satisfied for all the studied experimental trajectories.

The inequality $k_{\text {bal }}<\bar{k}$ limits how large the ballistic movement can be made restricting the value of $T_{m}$, see (16). The practical meaning of this inequality is that given the values of $a_{1}, t_{1}$, and $l$, the ballistic phase cannot achieve an arbitrary far point.

Now the desired function $f_{\text {bal }}\left(\tilde{P}_{m}, V_{m}\right)$ can be constructed. Choosing $k_{b a l}, \eta_{l}, \eta_{r}$ and $\phi(\eta)$ as suggested by Proposition 2 and recalling the definition (15), define

$$
f_{\text {bal }}(\eta):= \begin{cases}a_{2} & \text { for } \eta<\eta_{l}, \\ \phi(\eta) & \text { for } \eta_{l} \leq \eta \leq \eta_{r}, \\ a_{1} & \text { for } \eta>\eta_{r} .\end{cases}
$$

Note that the function $f_{b a l}(\eta)$ has the following properties: i) it is a continuous nondecreasing function, ii) it is strictly monotonic on $\left[\eta_{l}, \eta_{r}\right]$, and iii) $f_{\text {bal }}(0)=0$. The function $f_{b a l}(\eta)$ can be reconstructed based on an experimental trajectory data, and an example of such a function is given in Fig. 4.

To summarize, the model of the ballistic phase is given by (14), (15) and (21). Stability of this model is established by the following proposition. 
Proposition 3. Let Assumption 2 be satisfied and consider the system (14), (15) and (21) with $0<k_{\text {bal }}<\bar{k}$. All the trajectories of the system are bounded, and the equilibrium point $\tilde{P}_{m}=V_{m}=0$ is globally asymptotically stable.

Proof. Define $x(t):=\left[\tilde{P}_{m}(t), V(t)\right]^{\top}$. The system (14), (15), (21) can be rewritten as

$$
\begin{aligned}
\dot{x}_{1}(t) & =x_{2}(t), \\
\dot{x}_{2}(t) & =f_{\text {bal }}(\eta(t)), \\
\eta(t) & =-x_{1}(t)-k_{\text {bal }} x_{2}(t),
\end{aligned}
$$

which is a Lurie system. Note that due to the properties of $f_{b a l}(\eta)$ the following holds

$$
\eta f_{b a l}(\eta)>0 \text { for } \eta \neq 0 .
$$

Define a positive definite Lyapunov function

$$
W(x):=\frac{1}{2} x_{2}^{2}+\int_{0}^{\eta(x)} f_{b a l}(s) d s .
$$

Its time derivative is (the argument of time is omitted)

$$
\begin{aligned}
\frac{d W(x)}{d t} & =x_{2} \dot{x}_{2}+\dot{\eta} f_{b a l}(\eta) \\
& =x_{2} f_{b a l}(\eta)+\left(-x_{2}-k_{b a l} f_{b a l}(\eta)\right) f_{b a l}(\eta) \\
& =-k_{b a l} f_{b a l}^{2}(\eta) \leq 0 .
\end{aligned}
$$

Using standard Lyapunov arguments we conclude that $\eta(t), x_{2}(t)$, and so $x_{1}(t)$, are bounded, and $\eta(t) \rightarrow 0$ as $t \rightarrow \infty$. Note that the origin $x=0$ is the only invariant solution in the set $\{x \mid \eta(x)=0\}$. Applying LaSalle's Invariance Principle, the global asymptotic stability of the equilibrium point $x=0$ follows.

Propositions 1 and 3 ensure that as long as the model parameters are chosen to satisfy the assumptions, all trajectories generated by the proposed switched model will remain bounded and will converge to the target. From this point of view, these propositions may be considered as defining the set of feasible parameters.

\section{EXPERIMENTAL VALIDATION}

In this section, we present the experimental verification of the proposed switched model. From the considered dataset (see Section 2) we have chosen 7 trained participants and 3 trajectories for each of them, that is 21 trajectories in total. All the trajectories correspond to the constant-gain PTF (6) having 1.5 times velocity amplification. The mouse has 400 counts per inch, and the display admits resolution 98.5 pixels per inch. Therefore, the gain of the used PTF equals $g_{0}=1.5 \frac{98.5}{400}$, providing both counts-to-pixels scaling and velocity amplification. The measurements are performed with the sampling frequency $125 \mathrm{~Hz}$.

For each of the chosen trajectories we have identified parameters of the proposed model; see Table 1 for a list of the parameters. The identification was performed as follows. First, we separate the full movement into ballistic, switching and tracking phases, where the switching phase is defined as an interval with zero acceleration. Second, for the ballistic part we tune the parameters listed in the first row of Table 1 with a nonlinear optimization routine in order to minimize a quadratic approximation error cost function. Next, for the tracking part we use classic linear 
TABLE 1. Model parameters.

\begin{tabular}{cc}
\hline Ballistics & $a_{1}, a_{2}, t_{1}, t_{2}, g_{m}, k_{b a l}$ \\
\hline Switching & $P_{s w}, \delta_{s w}$ \\
\hline Tracking & $\tau_{p e r}, k_{v}, \gamma$ \\
\hline
\end{tabular}

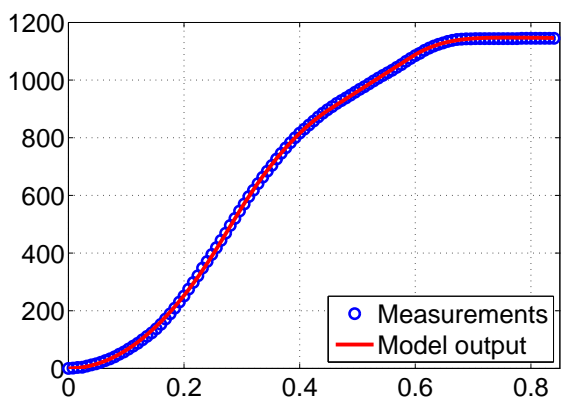

(A) Position (in pixels) versus time (in seconds).

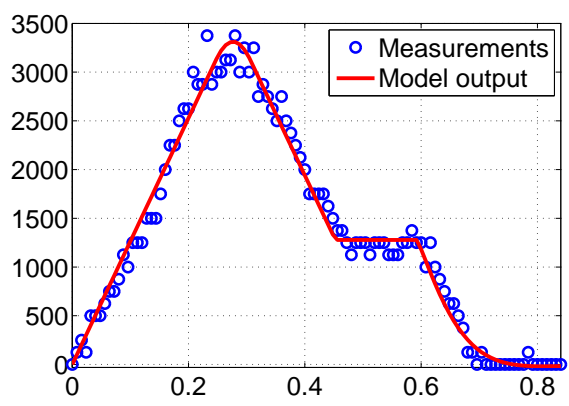

(в) Velocity (in pixels per second) versus time (in seconds).

Figure 5. Comparison of the proposed model output with the measured data, trajectory \#1.

system identification tools to tune the parameters listed in the last row of Table 1. Finally, we slightly adjust the switching time instance to match the velocity measured during the switching interval.

Figures 5, 6, and 7 present a comparison of the proposed model outputs with the measured data. Three types of trajectories are considered, where the first one corresponds to a typical pointing movement, the second trajectory type is a movement with an overshoot due to a long ballistic phase, and the third trajectory presents a relatively short ballistic movement. As it can be seen from the figures, all these different types of pointing motions are handled by the proposed model fitting well the measurements.

The same 21 trajectories are also used to identify and evaluate two alternative models: the ballistic model given by the minimum jerk principle [21], and a linear tracking model.

The minimum jerk principle claims that the ballistic cursor movement can be described with the curve $P_{c}(t)$ minimizing the total jerk cost functional

$$
J\left[P_{c}\right]:=\int_{0}^{t_{f}}\left(P_{c}^{(3)}(t)\right)^{2} d t
$$

where $t_{f}$ is the time instance when the movement stops. The Euler-Lagrange equation for (22) yields $\frac{d^{6}}{d t^{6}} P_{c}(t) \equiv 0$ and given the boundary conditions $P_{c}(0)=$ $P_{c}^{(1)}(0)=P_{c}^{(2)}(0)=0$ the desired curve is $P_{c}(t)=c_{5} t^{5}+c_{4} t^{4}+c_{3} t^{3}$. The constants $c_{3}, c_{4}$ and $c_{5}$ can be computed given the boundary conditions $P_{c}^{(1)}\left(t_{f}\right)=P_{c}^{(2)}\left(t_{f}\right)=$ 0 and $P_{c}\left(t_{f}\right)=T$, where the values $t_{f}$ and $T$ are specific for the exact trajectory. 


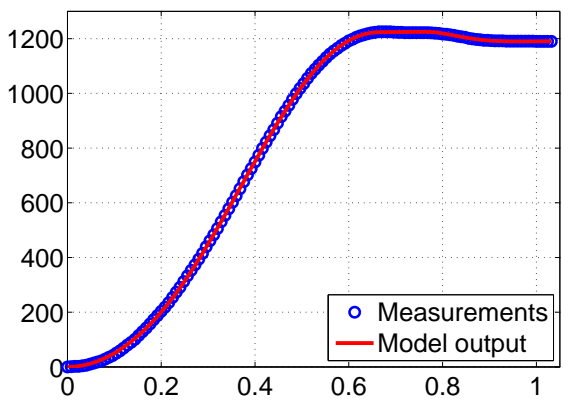

(A) Position (in pixels) versus time (in seconds).

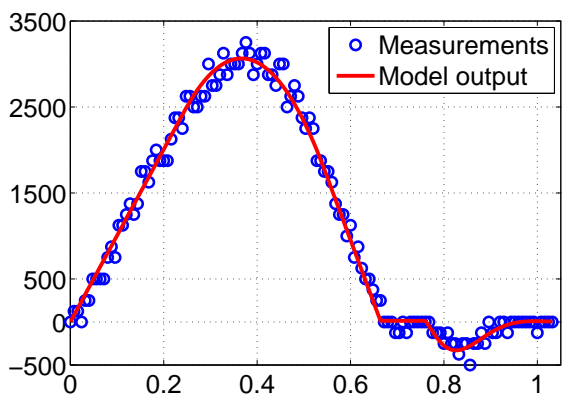

(B) Velocity (in pixels per second) versus time (in seconds).

Figure 6. Comparison of the proposed model output with the measured data, trajectory \#2.

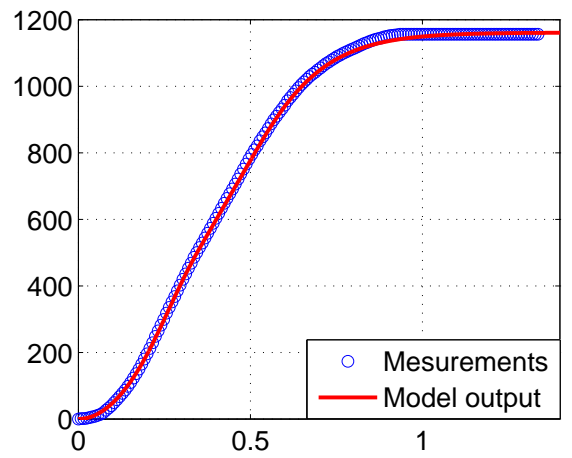

(A) Position (in pixels) versus time (in seconds).

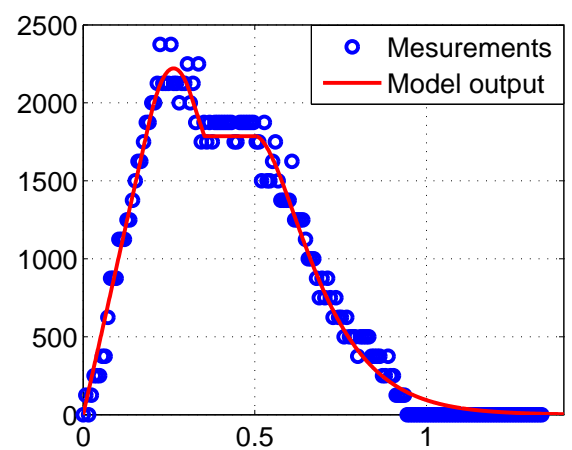

(B) Velocity (in pixels per second) versus time (in seconds).

Figure 7. Comparison of the proposed model output with the measured data, trajectory \#3 [36].

Straightforward computations yield

$$
P_{c}(t)=T\left(6\left(\frac{t}{t_{f}}\right)^{5}-15\left(\frac{t}{t_{f}}\right)^{4}+10\left(\frac{t}{t_{f}}\right)^{3}\right) .
$$

Fig. 8 presents comparison of the measured data with the ballistic model outputs for a typical pointing movement. One can see that the curve predicted with the minimum jerk ballistic model has significant deviations from the measured one, and this model cannot reconstruct the measured velocity profile.

As a linear tracking model we use the 3rd order transfer function from the input $T$ to the output $P_{c}$

$$
W_{t r c}(s):=\frac{a_{3} s+a_{0}}{s^{3}+a_{2} s^{2}+a_{1} s+a_{0}}
$$




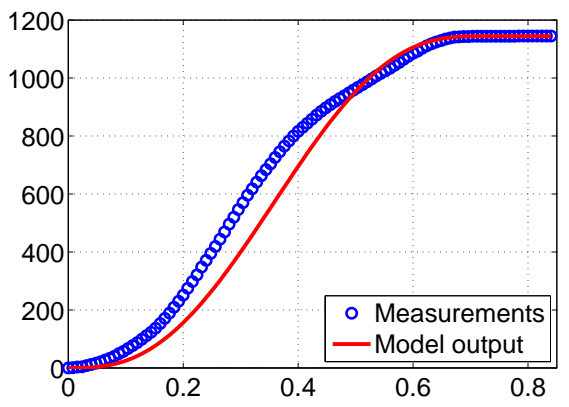

(A) Position (in pixels) versus time (in seconds).

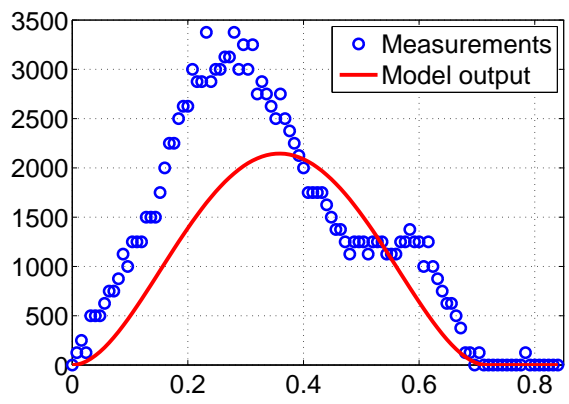

(B) Velocity (in pixels per second) versus time (in seconds).

Figure 8. Comparison of the ballistic model (23) output with the measured data, trajectory \#1.

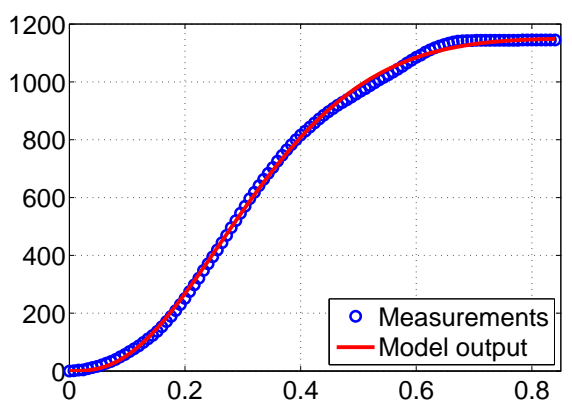

(A) Position (in pixels) versus time (in seconds).

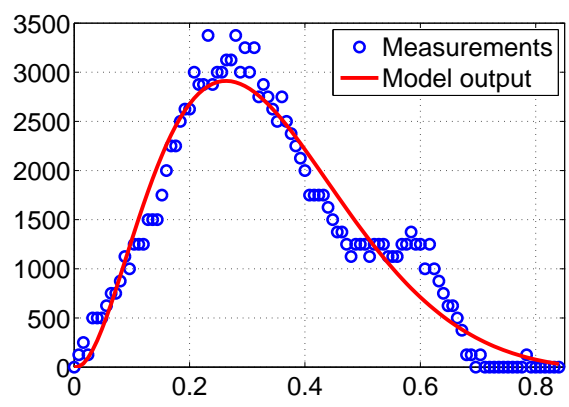

(B) Velocity (in pixels per second) versus time (in seconds).

Figure 9. Comparison of the linear tracking model (24) output with the measured data, trajectory \#1.

where the coefficients $a_{0}, a_{1}$ and $a_{2}$ are such that the transfer function is stable, and $a_{3} \geq 0$. These coefficients are tuned with standard linear system identification tools. It is worth noting that the general structure (24) can incorporate the tracking model (10), the crossover model [28], the VITE model [30] and the VITE-like model [32] with a constant-gain PTF. Fig. 9 presents comparison of the measured data with the linear tracking model outputs for a typical pointing movement. The curve predicted with the linear model has smaller deviations from the measurements than the ballistic model (23). However, as expected, the switching velocity profile cannot be reconstructed with the model (24).

For quantitative evaluation of the models we use the following metrics:

- Mean Absolute Error (MAE) is computed as

$$
M A E:=\frac{1}{N}\left\|P_{c, \text { meas }}-P_{c, p r e d}\right\|_{1}
$$


TABLE 2. Models performance comparision.

\begin{tabular}{lccc}
\hline & \multicolumn{3}{c}{ MAE } \\
& min. & max. & avg. \\
\hline Ballistic model & 46.9 & 242.9 & 109.1 \\
Linear model & 3.4 & 27.1 & 9.3 \\
Switched model & 1.1 & 6.1 & 3.0 \\
\hline \hline & \multicolumn{3}{c}{ ME } \\
& min. & max. & avg. \\
\hline Ballistic model & 119.8 & 621.7 & 280.0 \\
Linear model & 9.0 & 74.4 & 26.7 \\
Switched model & 4.3 & 26.5 & 11.1 \\
\hline \hline & \multicolumn{3}{c}{ NRMSE } \\
& min. & max. & avg. \\
\hline Ballistic model & 0.142 & 0.737 & 0.334 \\
Linear model & 0.010 & 0.083 & 0.029 \\
Switched model & 0.004 & 0.022 & 0.010 \\
\hline
\end{tabular}

where $N$ the number of measurements, $P_{c, \text { meas }}$ the measured cursor position, and $P_{c, \text { pred }}$ the cursor position predicted with a model under evaluation;

- Maximum Error (ME) is computed as

$$
M E:=\max \left|P_{c, \text { meas }}-P_{c, \text { pred }}\right| \text {; }
$$

- Normalized Root Mean Square Error (NRMSE) is computed as

$$
N R M S E:=\frac{\left\|P_{c, \text { meas }}-P_{c, \text { pred }}\right\|_{2}}{\left\|P_{c, \text { meas }}-\operatorname{mean}\left\{P_{c, \text { meas }}\right\}\right\|_{2}} .
$$

This value varies from zero to infinity, where zero means perfect fit, and if $N R M S E$ equals to 1 , then $P_{c, p r e d}$ matches $P_{c, \text { meas }}$ no better than a straight line.

These metrics are computed for each of the chosen 21 trajectories, the minimum, maximum and average values among these trajectories are given in Table 2 for the ballistic model (23), linear tracking model (24), and for the proposed switched model given in Fig. 2. The histograms of the MAE, ME and NRMSE metrics are shown in Figs. 10-12. The results illustrate that the proposed switched model significantly outperforms ballistic and linear ones.

\section{CONCLUSIONS}

In this work, the problem of modeling a pointing task with a computer mouse was studied. Based on the Optimized Initial Impulse model [15] and on the Surge model [33], the pointing movement was separated into three distinct steps: a ballistic phase, followed by a commutation phase with no acceleration, finished with a tracking phase. The obtained model is then a switching system. In the first stage, there is no visual guidance to the user, only a sensorimotor feedback is available, and a nonlinear system of Lurie form is used to model this part. The commutation phase happening when the approach of the final cursor position is perceived by the user, it triggers the switching to the tracking phase. In this last phase, thanks to the visual perception of the cursor position by the user, an extended VITE model [30] 


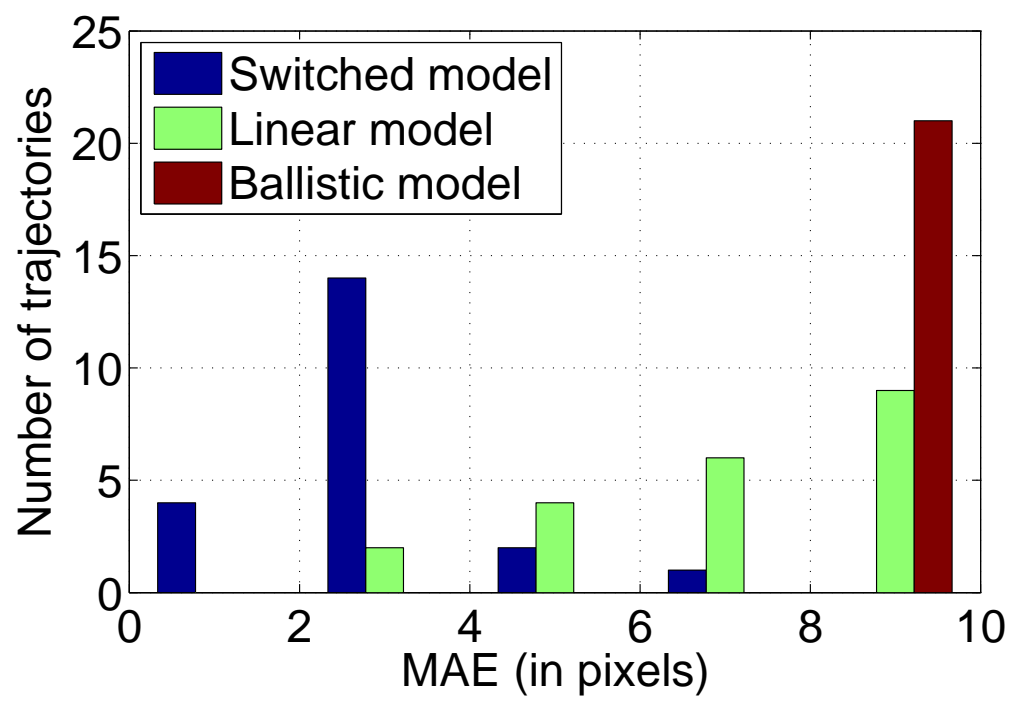

Figure 10. Histogram of the $M A E$ metric.

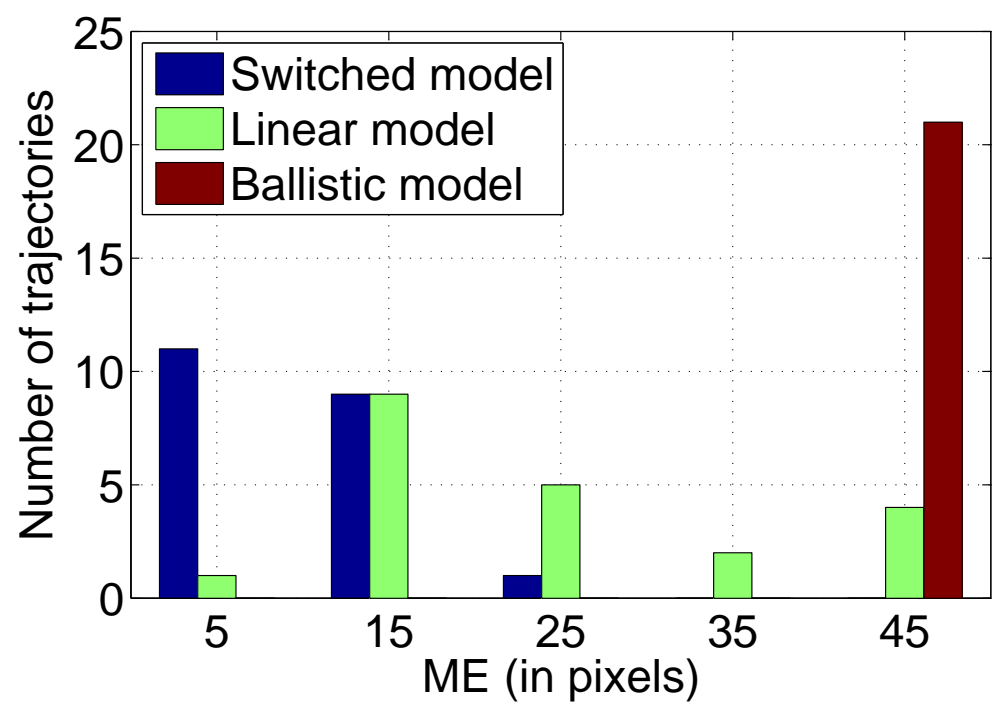

Figure 11. Histogram of the $M E$ metric.

is used to model the tracking dynamics (a linear filter is applied to model the visual perception). We have shown that both ballistic and tracking dynamics are globally asymptotically stable under some established mild conditions. In this note, only trained users were considered, so each phase was activated only once, therefore the problem of instability caused by commutation was not analyzed here.

A series of experiments were performed to validate the model showing that it fits well different types of pointing movements and outperforms pure ballistic and tracking models. 


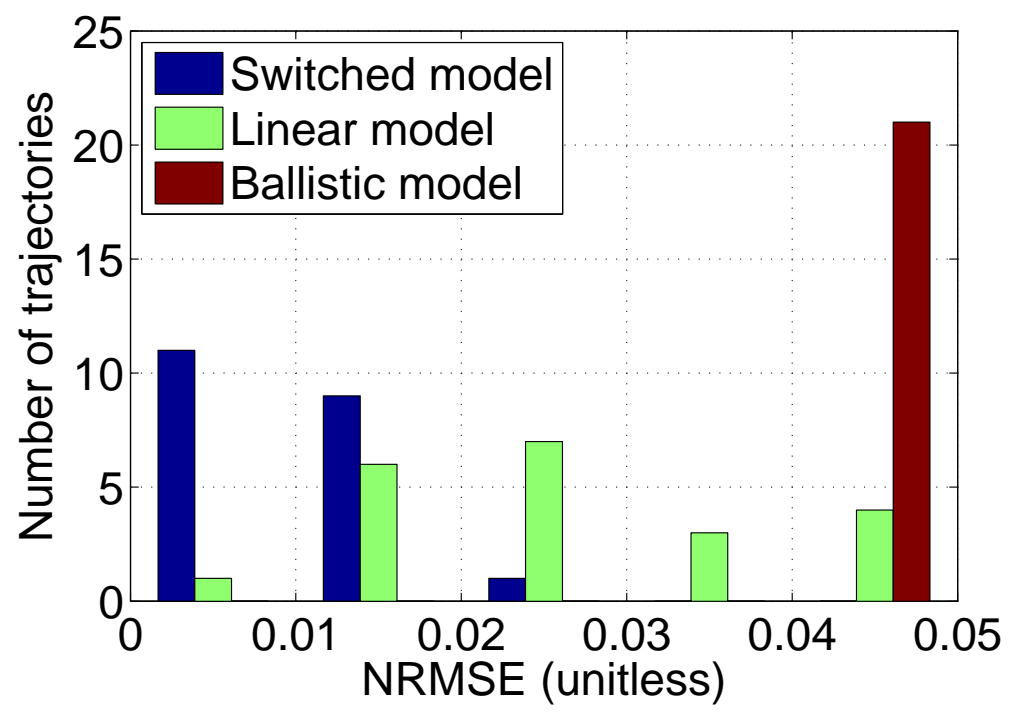

FigURE 12. Histogram of the NRMSE metric.

This first dynamical model for pointing tasks presented in our work, opens naturally new research directions. In the paper, the experimental validation has been performed for static-gain PTFs only. More realistic PTFs depending both on cursor velocity and position are an expected extension of our work. In addition, another extension would include the analysis of non-adapted users behavior that will naturally demand an extended stability analysis in the presence of switching. Moreover, the effect of delays on the closed-loop stability and the pointing task performance itself should be analyzed.

Another interesting research direction is to consider the proposed model from the biomechanical prospective and compare it with the dynamic models for wrist motions $[38,39]$ to see possible connections, e.g. velocity and acceleration limitations.

Finally, an automatic tuning of the interface to match the user type should be studied in order to identify users in terms of their experience by applying the proposed model. Further works may also be directed towards the proposition of a somehow optimal PTF design given a user model, and the extension of the approach to other pointing devices.

\section{REFERENCES}

[1] Casiez G, Roussel N. No more bricolage! Methods and Tools to Characterize, Replicate and Compare Pointing Transfer Functions. In: Proceedings of the 24th annual ACM symposium on User interface software and technology - UIST '11. Santa Barbara, CA, USA; 2011. p. 603.

[2] Casiez G, Vogel D, Balakrishnan R, Cockburn A. The Impact of Control-Display Gain on User Performance in Pointing Tasks. Human-Computer Interaction. 2008;23(3):215-250.

[3] Lank E, Cheng YCN, Ruiz J. Endpoint prediction using motion kinematics. In: Proceedings of the SIGCHI conference on Human factors in computing systems (CHI 07). San Jose, California, USA; 2007. p. 637-646.

[4] Balakrishnan R. "Beating" Fitts' law: Virtual enhancements for pointing facilitation. International Journal of Human Computer Studies. 2004;61(6):857-874. 
[5] McGuffin M, Balakrishnan R. Acquisition of Expanding Targets. In: Proceedings of the SIGCHI Conference on Human Factors in Computing Systems (CHI 02). Minneapolis, Minnesota, USA; 2002. p. 57-64.

[6] Cai Z, Shen C, Guan X. Mitigating Behavioral Variability for Mouse Dynamics: A Dimensionality-Reduction-Based Approach. IEEE Transactions on Human-Machine Systems. 2014;44(2):244-255.

[7] Hersch M, Billard AG. A biologically-inspired controller for reaching movements. In: Proceedings of the First IEEE/RAS-EMBS International Conference on Biomedical Robotics and Biomechatronics. Pisa, Italy; 2006. p. 1067-1072.

[8] Karimoddini A, Lin H. Hierarchical Hybrid Symbolic Robot Motion Planning and Control. Asian Journal of Control. 2014;17(1):23-33.

[9] Zhang ZQ, Ji LY, Huang ZP, Wu JK. Adaptive Information Fusion for Human Upper Limb Movement Estimation. IEEE Transactions on Systems, Man, and Cybernetics - Part A: Systems and Humans. 2012;42(5):1100-1108.

[10] Santiago DD, Slawiñski E, Mut VA. Stable Delayed Bilateral Teleoperation of Mobile Manipulators. Asian Journal of Control. 2017;19(3):1140-1152.

[11] Fitts PM. The information capacity of the human motor system in controlling the amplitude of movement. Journal of Experimental Psychology. 1954;47(6):381-391.

[12] MacKenzie IS. Fitts' law as a research and design tool in human-computer interaction. Human-Computer Interaction. 1992;7(1):48.

[13] Schmidt Ra, Zelaznik H, Hawkins B, Frank JS, Quinn JT. Motor-output variability: a theory for the accuracy of rapid motor acts. Psychological review. 1979;47(5):415-451.

[14] Crossman ER, Goodeve PJ. Feedback control of hand-movement and Fitts' Law. The Quarterly journal of experimental psychology Section A, Human experimental psychology. 1983;35(Pt 2):251-278.

[15] Meyer DE, Abrams Ra, Kornblum S, Wright CE, Smith JE. Optimality in human motor performance: ideal control of rapid aimed movements. Psychological review. 1988;95(3):340370.

[16] Chen Y, Hoffmann ER, Goonetilleke RS. Structure of Hand/Mouse Movements. IEEE Transactions on Human-Machine Systems. 2015;45(6):790-798.

[17] Senanayake R, Goonetilleke RS, Hoffmann ER. Targeted-Tracking With Pointing Devices. IEEE Transactions on Human-Machine Systems. 2015;45(4):431-441.

[18] Almanji A, Payne AR, Amor R, Davies TC. A Nonlinear Model for Mouse Pointing Task Movement Time Analysis Based on Both System and Human Effects. IEEE Transactions on Neural Systems and Rehabilitation Engineering. 2015;23(6):1003-1011.

[19] Gan KC, Hoffmann ER. Geometrical conditions for ballistic and visually controlled movements. Ergonomics. 1988;31(5):829-39.

[20] Oel P, Schmidt P, Schmitt A. Time prediction of mouse-based cursor movements. Proceedings of Joint AFIHM-BCS Conference on Human-Computer Interaction IHM-HCI. 2001;2:37-40.

[21] Hogan N. An organizing principle for a class of voluntary movements. The Journal of neuroscience : the official journal of the Society for Neuroscience. 1984;4(11):2745-2754.

[22] Richardson MJE, Flash T. Comparing smooth arm movements with the two-thirds power law and the related segmented-control hypothesis. The Journal of neuroscience : the official journal of the Society for Neuroscience. 2002;22(18):8201-8211.

[23] Berret B, Darlot C, Jean F, Pozzo T, Papaxanthis C, Gauthier JP. The inactivation principle: Mathematical solutions minimizing the absolute work and biological implications for the planning of arm movements. PLoS Computational Biology. 2008;4(10):1 - 25.

[24] Berret B, Gauthier JP, Papaxanthis C. How humans control arm movements. Proceedings of the Steklov Institute of Mathematics. 2008;261:44-58.

[25] Berret B, Chiovetto E, Nori F, Pozzo T. Evidence for composite cost functions in arm movement planning: An inverse optimal control approach. PLoS Computational Biology. $2011 ; 7(10): 1-18$.

[26] Todorov E, Jordan M. Optimal feedback control as a theory of motor coordination. Nature neuroscience. 2002;5(11):1226-1235.

[27] Todorov E. Optimality principles in sensorimotor control. Nature neuroscience. 2004;7(9):907-15.

[28] McRuer DT, Jex HR. A Review of Quasi-Linear Pilot Models. Human Factors in Electronics, IEEE Transactions on. 1967;HFE-8(3):231-249. 
[29] Müller J. Dynamics of Pointing with Pointer Acceleration. In: Human-Computer Interaction - INTERACT 2017. Springer International Publishing; 2017. p. 475-495.

[30] Bullock D, Grossberg S. Neural dynamics of planned arm movements: emergent invariants and speed-accuracy properties during trajectory formation. Psychological review. 1988;95(1):49-90.

[31] Wolpert DM, Ghahramani Z, Jordan MI. An internal model for sensorimotor integration. Science. 1995;269(5232):1880.

[32] Varnell P, Zhang F. Characteristics of Human Pointing Motions with Acceleration. In: 54th Conference on Decision and Control. Osaka, Japan; 2015. p. 5364-5369.

[33] Costello RG. The Surge Model of the Well-Trained Human Operator in Simple Manual Control. IEEE Transactions on Man-Machine Systems. 1968;9(1):2-9.

[34] Maggio M, Papadopoulos AV, Leva A. On the Use of Feedback Control in the Design of Computing System Components. Asian Journal of Control. 2012;15(1):31-40.

[35] Müller J, Oulasvirta A, Murray-Smith R. Control Theoretic Models of Pointing. ACM Trans Comput-Hum Interact. 2017;24(4):27:1-27:36.

[36] Aranovskiy S, Ushirobira R, Efimov D, Casiez G. Modeling pointing tasks in mouse-based human-computer interactions. In: Decision and Control (CDC), 2016 IEEE 55th Conference on. IEEE; 2016. p. 6595-6600.

[37] Khalil HK. Nonlinear Systems. New Jersey: Prentice Hall; 2002.

[38] Ramsay JW, Hunter BV, Gonzalez RV. Muscle moment arm and normalized moment contributions as reference data for musculoskeletal elbow and wrist joint models. Journal of Biomechanics. 2009;42(4):463 - 473.

[39] Lemay MA, Crago PE. A dynamic model for simulating movements of the elbow, forearm, and wrist. Journal of biomechanics. 1996;29(10):1319-1330.

Stanislav Aranovskiy is with Equipe Automatique (AUT), CentraleSupélec - IEtr, CS 47061 Avenue de la Boulaie, 35576 Cesson-Sévigné, France, and with Department of Control Systems and Informatics, Itmo University, Kronverkskiy Pr., 49, St. PetersBURG, 197101, Russia [stanislav.aranovskiy@centralesupelec.fr], RosANE UsHIROBIRA and Denis Efimov are with InRIa, Univ. Lille, CNRS, UMR 9189 - CRIStAL, F-59000 Lille, France [rosane.ushirobira@inria.fr, Denis.Efimov@inria.fr], GÉRY CASIEZ IS with with CRIStAL (UMR CNRS 9189), Villeneuve D'AscQ, France and with InRIa, Loki team, 40 avenue Halley, 59650 Villeneuve D'AscQ, France [gery.casiez@univ-lille.fr] 